\title{
Cloud-Based Knowledge Management Framework for Decision Making in Higher Education Institutions
}

\author{
Muhammad Younas ${ }^{1,2, *}$, Ahmad Shukri Mohd Noor ${ }^{2}$ and Muhammad Arshad ${ }^{1}$ \\ ${ }^{1}$ Jazan University, Jazan, 82182, Saudi Arabia \\ ${ }^{2}$ Universiti Malaysia Terengganu, Kuala Terengganu, 21030, Malaysia \\ ${ }^{*}$ Corresponding Author: Muhammad Younas. Email: mmuhammad@jazanu.edu.sa \\ Received: 05 March 2021; Accepted: 24 May 2021
}

\begin{abstract}
The education sector is now necessitated to align itself with the latest innovative technological trends in the industry to be competitive while transforming database management practices. The Higher Education Institutions (HEIs) are currently using cloud-based applications at varied implementation levels while others are following the shifting trends. On-demand availability of consolidated information is a critical success factor to perform decision-making activities; as a result, quite a bit of research work has been performed in this realm. The study is aimed to propose an efficient framework that is capable of extracting the desired information from an educational setup intended to be used for decision-making purposes over cloud infrastructure. The framework focuses on addressing all the desired queries to be used for administrative and decision-making purposes. Further, different institutions will be benefited from enhanced and efficient decision-making with high accuracy and to boost inter and intra institutions' knowledge sharing tasks. In conclusion, on the premise of the decision-making architecture, a cloud-based knowledge management reference prototype framework has been created and implemented to verify the outcomes for one of the public universities in the Kingdom of Saudi Arabia (KSA).
\end{abstract}

Keywords: Knowledge management; decision making; cloud computing; higher education institutions; knowledge management system

\section{Introduction}

An organization's data management is highly important and is becoming ever more complex due to incessant increments in higher volume and processing needs. Thus, the required knowledge is extracted in terms of information in ready form from raw data [1]. The required information extraction from massive data is yet another challenging task for an organization while different types of knowledge extraction techniques are used for this purpose. State-of-the-art and expensive equipment is required to perform the knowledge extraction process to meet the needs of the organization's goal. The process of extracting information when required at a given point in time is called knowledge management (KM) [2].

This work is licensed under a Creative Commons Attribution 4.0 International License, which permits unrestricted use, distribution, and reproduction in any medium, provided the original work is properly cited. 
Advancements in technology make it necessary for organizations to adopt the latest technology to meet the contemporary needs of the technological advancement. The cost has always remained a big concern for an organization and is considered to be a barrier for the latest technology adoption along with another list of stoppers. HEIs are ideal for KM implementation, and institutional success can be amplified with the KM in terms of social and educational values leading to the ability to make a good decision using the available knowledge [2]. On the other hand, HEIs are also struggling to keep up with the innovative trends of the ever-changing technology which makes it indispensable to adopt the latest technology to overcome the current challenges.

Cloud computing (CC) has become one of the pioneering and leading technologies available today which aid in reducing cost with pay-as-you-go services and reduce management effort in terms of time and resource supervision [3]. CC is an attractive stake for any organization due to its ease of use while the end-user gets to use only those services which are authorized by the service provider. They are oblivious to and least concerned with background information and processing. CC can be defined as an infrastructure where data storage and processing occurs external to the user's devices that pass through these databases [4]. Unintentional use of CC services like Dropbox, Facebook, Gmail, Office 365, Amazon web services etc. in our daily lives is common. The use of KM in different decision-making activities is common within the organization while its performance can be enhanced dramatically with the use of advanced technology like $\mathrm{CC}$ which assists in effective decision making. The decision-making activity success in any HEI is directly impacted by the implementation of an advantageous architectural design and knowledge extraction technique which plays an important role thereof.

The purpose of this research is to create a framework for university management to increase and enhance the management-related process with on-demand knowledge availability. The proposed architectural framework intended to be used by the end-user is a combination of three main stages, namely, knowledge extraction, knowledge storage and visualization. In this framework, the knowledge is extracted and made available to the management when required by the management as per the organizational needs based on the post-application of business logic. This will help the organization perform different decision-making processes as well as share knowledge within and outside the organization.

The paper is organized into eight sections, namely, Section 2 covers the literature review, Section 3 presents the methodology, Section 4 demonstrates the proposed framework in detail, Section 5 describes the reference architecture of one of KSA's public universities, Section 6 displays the result and discussion, Section 7 highlights the challenges and future work and, finally, the conclusion with remarks is mentioned in Section 8.

\section{Literature Review}

A substantial amount of research work has been accomplished by many scholars in the KM field who have recognized its importance. In this present study, to better grasp the subject of KM outcomes coupled with $\mathrm{CC}$, the authors have attempted to study the preceding literature that has significantly contributed to establishing a knowledge management framework. The study endeavors to realize the importance of the framework in decision-making while recommending its application. A cloud-based knowledge management framework was proposed expending a knowledge engineering approach with Software as a Service (SaaS) over a secluded cloud [5]. The proposed framework comprises two phases; namely, analysis, integration of data and framework design. The four characteristics of the proposed framework include retrieval, storage, sharing and knowledge publishing. The framework uses a knowledge engineering approach to fulfill the knowledge management characteristics for distributing, reusing and transferring knowledge. Not much detail or methodology is available to demonstrate knowledge saving and updating mechanisms over the cloud according to the author. Based on a study on the knowledge 
management process through a cloud computing-based approach conducted by Chikhi et al. [6], the author proposed a complete framework to attain a cloud computing-based knowledge management infrastructure while taking into account all its sub-processes (knowledge acquisition, knowledge spotting, knowledge utilization, knowledge updating etc.). The proposed framework attempts to shift all individual processes over the cloud and use it as a service. The Knowledge Management as a Service (KMaaS) will be used for the Knowledge model, employee, decision-maker and knowledge holders, respectively. The proposed framework has not yet been implemented to verify the result and monitor its performance.

Rahamathulla [7] proposed in a cloud-based framework for health care data management that used the cloud services from the top three service providers for data management in health care. This supports multisource data uploading as the main feature that meets the health industry's needs. The framework presents the layer-wise data handling with the appropriate cloud service. The author has used the amazon cloud service as a reference by constructing the architecture for cloud-based health care. The research focused on multisource data uploading issues combined with big health care data storage over the cloud. This study is limited to the health industry and mentions no criteria for an appropriate cloud service selection to meet the health care-related needs of an organization.

Alhammadi [8] proposed a decision-making framework for the adoption of cloud-based knowledge management which consists of three decision-making levels, i.e., the strategic level, cloud deployment selection level and operational level. Each stage provides comprehensive information and results for the decision-maker while narrowing the result by ending the desired result set. According to the author, the prime objective of this study is to investigate the problems, implement the benefits of the study, and encourage its acceptance for cloud adoption in technology in developing nations. However, this study is not fit for technologically advanced countries and, thus, proposes its application to less technologically developed countries only such that it can verify the differences. Tab. 1 shows the related work summary.

Table 1: Literature summary

\begin{tabular}{|c|c|c|c|c|c|c|}
\hline $\begin{array}{l}\text { Reference } \\
\text { Paper }\end{array}$ & Title & $\begin{array}{l}\text { Framework/ } \\
\text { Model }\end{array}$ & $\begin{array}{l}\text { Knowledge } \\
\text { Management }\end{array}$ & $\begin{array}{l}\text { Cloud } \\
\text { Computing }\end{array}$ & $\begin{array}{l}\text { Cloud } \\
\text { Service }\end{array}$ & Domain \\
\hline $\begin{array}{l}\text { Anupan et al. } \\
\text { [5] }\end{array}$ & $\begin{array}{l}\text { A framework for a } \\
\text { knowledge management } \\
\text { system in a cloud computing } \\
\text { environment using a } \\
\text { knowledge engineering } \\
\text { approach }\end{array}$ & $\begin{array}{l}\text { KM } \\
\text { Framework }\end{array}$ & $\Pi$ & $\Pi$ & $\begin{array}{l}\text { SaaS } \\
\text { (Private } \\
\text { Cloud) }\end{array}$ & $\begin{array}{l}\text { Knowledge } \\
\text { Management }\end{array}$ \\
\hline $\begin{array}{l}\text { Chikhi et al. } \\
\text { [6] }\end{array}$ & $\begin{array}{l}\text { Knowledge Management } \\
\text { Process Through a Cloud } \\
\text { Computing Based Approach }\end{array}$ & $\begin{array}{l}\text { Framework } \\
\text { to achieve } \\
\text { KM } \\
\text { Infrastructure }\end{array}$ & $\Pi$ & $\Pi$ & $\begin{array}{l}\text { KMaaS } \\
\text { IaaS } \\
\text { KaaS }\end{array}$ & $\begin{array}{l}\text { Knowledge } \\
\text { Management }\end{array}$ \\
\hline $\begin{array}{l}\text { Rahamathulla } \\
\text { et al. [7] }\end{array}$ & $\begin{array}{l}\text { Proposed Cloud-based } \\
\text { Healthcare data management } \\
\text { Framework }\end{array}$ & $\begin{array}{l}\text { Data } \\
\text { Management } \\
\text { Framework }\end{array}$ & $\Pi$ & $\Pi$ & & Healthcare \\
\hline $\begin{array}{l}\text { Alhammadi } \\
\text { [8] }\end{array}$ & $\begin{array}{l}\text { Proposed a } 3 \text { level decision- } \\
\text { making framework for cloud } \\
\text { adoption in knowledge } \\
\text { management. }\end{array}$ & $\begin{array}{l}\text { Cloud } \\
\text { adoption } \\
\text { Decision } \\
\text { making } \\
\text { Framework }\end{array}$ & $\Pi$ & $\Pi$ & & $\begin{array}{l}\text { Knowledge } \\
\text { management } \\
\text { cloud } \\
\text { computing }\end{array}$ \\
\hline $\begin{array}{l}\text { Al-Qurishi } \\
\text { et al. [9] }\end{array}$ & $\begin{array}{l}\text { Proposed a framework of } \\
\text { knowledge management as a } \\
\text { service over a cloud platform }\end{array}$ & $\begin{array}{l}\text { Knowledge } \\
\text { Management } \\
\text { Framework }\end{array}$ & $\Pi$ & $\Pi$ & KMaaS & $\begin{array}{l}\text { Knowledge } \\
\text { management } \\
\text { as a service }\end{array}$ \\
\hline
\end{tabular}


Al-Qurishi et al. [9] proposed a framework that uses KM as a service over CC. The proposed framework consists of six components, which are the User, KM domain registration, KM service directory, Resource allocation manager, cloud manager and, finally, monitoring and payment. The user connects to the subscribed $\mathrm{KM}$ domain service while the $\mathrm{KM}$ administrator is responsible for maintaining business rules and logic implementation according to a specific domain. The KM service directory is considered as a main part of the framework according to the author and is responsible for interfacing between different users and cloud services. Even though, the proposed framework lacks its practical implementation at present since it is a prototype framework and its performance is yet to be evaluated.

Liao et al. [10] proposed a three-level conceptual framework for the knowledge management system (KMS) using CC. According to the author, the KM Strategic and plan component will identify the information that is required to be extracted for the KM and align it with the organizational needs. KMS Infrastructure is responsible for the required services used by $\mathrm{CC}$ and $\mathrm{KM}$ Applications which will be used for the knowledge flow required and controlled by the organization.

A conceptual framework was proposed by Baharuddin et al. [11] for the dynamic decision-making process. The proposed framework uses multisource data and extracts relevant information that can be used for dynamic decision-making. de Oliveira et al. [12] accentuated the importance of information technology tools for KM and its impact across different levels of an organization in decision making. The outcome yields a cost reduction factor upon the appropriate implementation. The authors further discuss different Management levels incorporated with Information technology including DSS (Decision Support System) and GDSS (Group Decision Support System).

By combined implementation of Knowledge Discovery (KD) and Intelligent Decision Support System (IDSS), the Intelligent Human Resource Information System (I-HRIS) framework was proposed by Masum et al. [13]. The proposed framework consists of three parts; an input system, decision making and ten HR modules as an output. According to Feldman [14], business decisions based on available data should go through the analytic analysis. An Education Plan is defined for the customer who follows the DMN (Decision Model and Notation). The Educational plan consists of predefined steps including Decision, Business Glossary, Business Model, Test cases and IT Integration. An educational model which uses CC was proposed by Ahmed et al. [15]. The proposed model gives access to the faculty members, students, as well as their parents to obtain real-time information about their child/student. With the inclusion of an Expert as a Service (EaaS), a cloud-based framework was designed by Navimipour et al. [16]. The proposed framework is a four-layered architecture. The outcomes of the expert cloud has yielded improved human resource utilization, a higher level of customer satisfaction and reduced response time.

The object of this paper has been to introduce a framework that increases knowledge sharing and collaboration in HEIs. The paper examines the idea of knowledge management in HEIs and presents a systematization of knowledge practices and devices to link individuals (understudies, teachers, researchers, secretariat staff, external elements) and promote knowledge sharing across several key cycles and administrations in a higher education institution, for example, the research measures, learning cycles, understudy and alumni administrations, administrative administrations and measures, and strategic planning and management. The framework purposed in this paper aims to improve the knowledge practices and cycles that facilitate a climate and a culture of knowledge collaboration and sharing, and a revelation that ought to characterize an institution of higher education.

\subsection{Knowledge Management}

Knowledge is a ready form of valuable information that is most relevant, contextual and dynamic by nature. The required information extraction technique from available raw data is called KM [2]. Knowledge can be classified into two types, which are explicit and implicit knowledge [17]. 
Raj [18] and Anand et al. [19] clustered knowledge into explicit and tacit as the main types. Hence, in general, knowledge can be categorized into three types including Explicit Knowledge (easily recorded, stored and shared), implicit knowledge (experience/skill gained with job switching) and tacit knowledge (personal experience). Tacit knowledge is difficult to record, and transferring tacit knowledge into explicit knowledge is one of the main KM tasks [20].

There are four communication modes between tacit and explicit knowledge also called knowledge conversation. These were first introduced by Nonaka and Takeuchi (1996) in the SECI (socialization, externalization, combination, internalization) Model and further explained by Qwaider [21] and Amine et al. [22]. The four modes of communication include Tacit to Tacit (Socialization), Tacit to explicit (Externalization), Explicit to Tacit (internalization) and Explicit to Explicit (Combination).

KM has gained importance and increased since knowledge is considered to be an integral part of the organization. Improving organizational Learning capacity is an essential part of KM [23]. It also becomes essential for information management platform perfection. Organizations can extend their competitions and get more benefits by getting, saving, settling, transforming, innovating and sharing knowledge as a part of the KM process [24].

\subsection{Cloud Computing (CC)}

The pay-as-you-go service feature of CC makes it stand out and is the most preferred choice of the latest technology among enterprises. While $\mathrm{CC}$ is considered to be the largest hosted internet-based service platform, it also defines itself by offering all the data processing and storage activities external to the user's machine [25]. CC has been illustrated as a new IT delivery model [26]. Before the pioneering advent of $\mathrm{CC}$, data security and sensitivity always remained the foremost concern out of all the big concerns for any organization, which also kept them from outsourcing their confidential resources [25].

The four types of CC categories are also called the deployment models [25]. These include the Private and Public Cloud, and Community and Hybrid Cloud, respectively. The CC offers three service models, namely, SaaS (Software as a service), also called software on-demand service; PaaS (Platform as a service) and IaaS (Infrastructure as service). The five key CC characteristics of the model include ondemand self-service, wide access to the network, Rapid scalability and elasticity, Resource multioccupancy and pooling and, finally, measured service.

\subsection{Decision Making Using Knowledge Management}

Making the right decision at the right time with accurate information can increase an organization's success and raise its confidence level for future business. Many authors have defined the decision-making process in their own way and agreed that it relates to choosing the best one from the multiple available alternatives.

A Decision-Making System in the education sector is essential for top management to perform daily decision-making activities with on-demand data availability and accuracy. Decision making is believed to be an art for managers and is used to achieve good quality decisions [27]. The decision-making process is considered as the core of a management activity due to its key managerial activity [28]. Decision-makers gain knowledge experiences from previous decisions and use them to solve co-related problems with a better approach. The decision-making process was initially described by Delibašić et al. [29]. A decision support system depends on different techniques with strong analytical data. The action decision is supported by the direction depending on the decision management techniques to enable business decisions [14]. 
Decision-making data should go through a proper analysis before being applied in decision making to make the data trustworthy for higher management. The educational opportunities and research works accomplished by different learners' experiences have attempted to fill the educational gap worldwide [30].

\subsection{Cloud-Based Knowledge Management}

$\mathrm{KM}$ is a replica of other management processes including time management and self-management, respectively. It is a function of the conditional knowledge usage based on "When" and "How". The technological implementation of $\mathrm{KM}$ is called KMS. KM has been appraised to the highest acknowledgment by both HEIs and their affiliates. It has just not only earned the stature of a high knowledge delivery platform but has also benchmarked itself for all the activities related to curricula design and knowledge sharing processes. $\mathrm{CC}$ is the fastest growing and most adaptive technology at present which has thereby attracted the attention of many organizations and enterprisers by offering ondemand services that meet the organizational needs of the businesses at low operating costs. HEIs are being forced to adopt technological changes and stand in the list of organizations ready to adopt the latest technology to meet the latest needs and growth.

The combination of KM and $\mathrm{CC}$ can be a significant contributing factor for organizational success in terms of performance. The relationship between $\mathrm{KM}$ and $\mathrm{CC}$ could be considered to be ideal under some circumstances. According to Chen [31] and Duan et al. [32], KM and CC have some common properties like data sharing, storing and analysis services that make $\mathrm{KM}$ an ideal example in $\mathrm{CC}$.

\subsection{Knowledge Management in University Environment}

The education sector is lacking innovative trends in technology as well as social and educational responsibilities to meet the modern educational needs and be aligned with the latest technology. Universities and other educational institutions are focusing on innovative teaching techniques by using their management models [15]. Technology contributes a vital role in the excellence of education. The most essential task of KM in HEIs is knowledge-creating and sharing. In such a setting, the knowledge is transferred from a researcher to the students via the teacher as the facilitator in the middle of an academic society.

Knowledge is created through different ways of research activities that are shared within the entire academic society and automatizing KM implementation across the university setting [33]. This University's KMS is composed of three modules, namely, the Research activity KM Module (RKM), Teaching activity KM Module (TKM) and University Management KM Module (UKM).

The IT Infrastructure and university portal are considered to act as a supporting module used by potential users including students, academic staff and other individuals who are active in academic university activities. The TKM module manages all knowledge relating to the teaching activities. The RKM module manages research-related knowledge activities carried out at the university. The UKM module is responsible for managing all administrative related activities.

\section{Methodology}

The research design methodology involved a mixed-method with qualitative and quantitative research approaches. To design a cloud-based knowledge management framework for decision making in a university setting the subsequent steps were followed.

\subsection{Target Population}

Twenty-eight corresponding Full-time faculty staff including the executives and decision-makers of Jazan University were the target population for this research. The population size was consciously 
selected subject to the nature of the information and availability. The selected staff was further grouped into two categories as shown in Tab. 2 below.

Table 2: List of the user population

\begin{tabular}{llll}
\hline Group Name & Group Code & Member Role/Position & Total \\
\hline Faculty & F & Faculty Member/Data management user/administrative staff & 18 \\
Administrative & A & President/Vice President/Assistant/vice president/Faculty Deans & 10 \\
\hline
\end{tabular}

Group F consisted of end-users who were engaged in data processing and management in a conventional system. Group A consisted of administrative users who were engaged in decision-making and performance evaluation.

\subsection{Real-Time Data Gathering and Analysis}

To create a prominent case, the research design involves the proposed framework in the real-time requirements gathering and analysis from the university project owned by the government of Saudi Arabia. The team comprised of the authors themselves was engaged in data gathering and analysis activities throughout all the stages. The team consisted of 28 (twenty-eight) respondents comprised of the university staff and executives, including two vice presidents and three assistant vice presidents. Primary chunks of information were gathered through focus group discussions, unstructured interviews and observations while some data were also collected by the review of various documents. It accounted for 60 days to accomplish the data gathering task.

\section{Proposed Framework}

KMS supports a variety of managerial tools, including query and reporting tools, data saving coupled with the inclusion of historical information and data consolidation for management analysis and decisionmaking. Managing all the existing data is not the sole task of the KMS, but rather managing the required knowledge and making it available to top management for decision-making purposes is also one of its tasks [34]. Data can be extracted from different sources, both internal and external to the organization. Data extraction from different sources and storage in one place is an uncommon practice rarely done by an organization [11]. CC defines itself as enabling everything on the cloud, including the infrastructure and different software-based levels of services offered by it. However, the authors argue that only extracted knowledge is to be saved on the cloud that can be used in decision making or other managerial tasks rather than uploading all organizational data to minimize risks of cloud data security breaches as well as minimize cost for the selective cloud services usage.

The proposed framework is a partial derivative of a project in one of the Saudi Public Universities and adopts the extended proposed framework described by Rahamathulla [7]. The main objective of the project is to design, develop and implement the KMS for university top-level management and decision-makers to provide the latest on-demand real-time information and consolidated report generation from multisource databases. The system should also be easily accessible anywhere at any time on all types of devices. The on-demand real-time bespoke reports will provide the desired range of queries and information, such as teaching load, student-teacher ratio, student statistical, university faculty information, blacklisted Faculty Members/Students, Dashboard and other managerial-related reports. The academic data are a mix of different formats including spreadsheets, CSV files and database tables/views. The data can be multisourced based on the level of organization and nature. Tab. 3 illustrates the comparison of the proposed with the available frameworks already described in the literature review. 
Table 3: Comparison of proposed vs. existing

\begin{tabular}{|c|c|c|c|c|c|}
\hline Attributes & $\begin{array}{l}\text { Proposed } \\
\text { Framework }\end{array}$ & $\begin{array}{l}\text { Cloud-Based } \\
\text { KM Using } \\
\text { Knowledge } \\
\text { Engineering } \\
\text { Approach } \\
\text { [5] }\end{array}$ & $\begin{array}{l}\text { Knowledge } \\
\text { Management Process } \\
\text { Through a Cloud } \\
\text { Computing Based } \\
\text { Approach } \\
\text { [6] }\end{array}$ & $\begin{array}{l}\text { cloud-based } \\
\text { framework for } \\
\text { healthcare data } \\
\text { management } \\
{[7]}\end{array}$ & $\begin{array}{l}\text { Expert Cloud: A Cloud- } \\
\text { based framework to share } \\
\text { the knowledge and skills } \\
\text { of human resources } \\
{[16]}\end{array}$ \\
\hline $\begin{array}{l}\text { Adopted } \\
\text { cloud service }\end{array}$ & PaaS & SaaS & KMaaS & (PaaS) & EaaS \\
\hline $\begin{array}{l}\text { Knowledge } \\
\text { updating }\end{array}$ & High & Low & Low & High & High \\
\hline $\begin{array}{l}\text { End-user } \\
\text { decision- } \\
\text { making } \\
\text { facilitation }\end{array}$ & High & low & Low & Medium & Low \\
\hline $\begin{array}{l}\text { Knowledge } \\
\text { retrieval }\end{array}$ & High & High & Medium & Medium & High \\
\hline $\begin{array}{l}\text { Response } \\
\text { time }\end{array}$ & Low & Medium & Medium & Low & Low \\
\hline $\begin{array}{l}\text { End-user } \\
\text { visualization }\end{array}$ & High & Low & Low & High & Medium \\
\hline $\begin{array}{l}\text { Accessibility } \\
\text { and Mobility }\end{array}$ & $\begin{array}{l}\text { High and } \\
\text { Medium }\end{array}$ & $\begin{array}{l}\text { Medium and } \\
\text { Low }\end{array}$ & Low & $\begin{array}{l}\text { High and } \\
\text { Medium }\end{array}$ & Medium and Low \\
\hline
\end{tabular}

The proposed framework consists of three main phases, which are knowledge extraction, knowledge storage and cloud service for end-users, respectively. This applies the extracted knowledge to perform different decision-making tasks. The first phase is the data extraction that applies the ETL technique used to pull the required information from the academic setup. The extracted information from the source data is the result of appropriate analysis and business logic implementation. Once the required information is extracted, it populates in the knowledge database during the second phase of the framework. The final phase is the access to the stored information using the cloud service. The end-users of the proposed framework are the top management and decision-makers from the education sector. Fig. 1 shows a cloudbased knowledge management framework for decision-making in the education sector. The details of each phase are discussed in the section below.

\subsection{Knowledge Extraction}

The extraction of knowledge from the source data is the first phase. The source data can be an internal (database), external (file) or combination of both with a multi-source option. This is a very important phase since all other processing is dependent on the data extracted from the knowledge extraction phase. The required information will be extracted from the data source by the application of business logic and ETL (Extraction, Transformation, Loading) to be used for this job. ETL is referred to as the method of data replication from a range of sources into the destination system [35]. Henceforth, this process is commonly used for multisource inter-data transfers and is considered to be the key factor in building a data warehouse since all the data sources must undergo the ETL process [36]. 


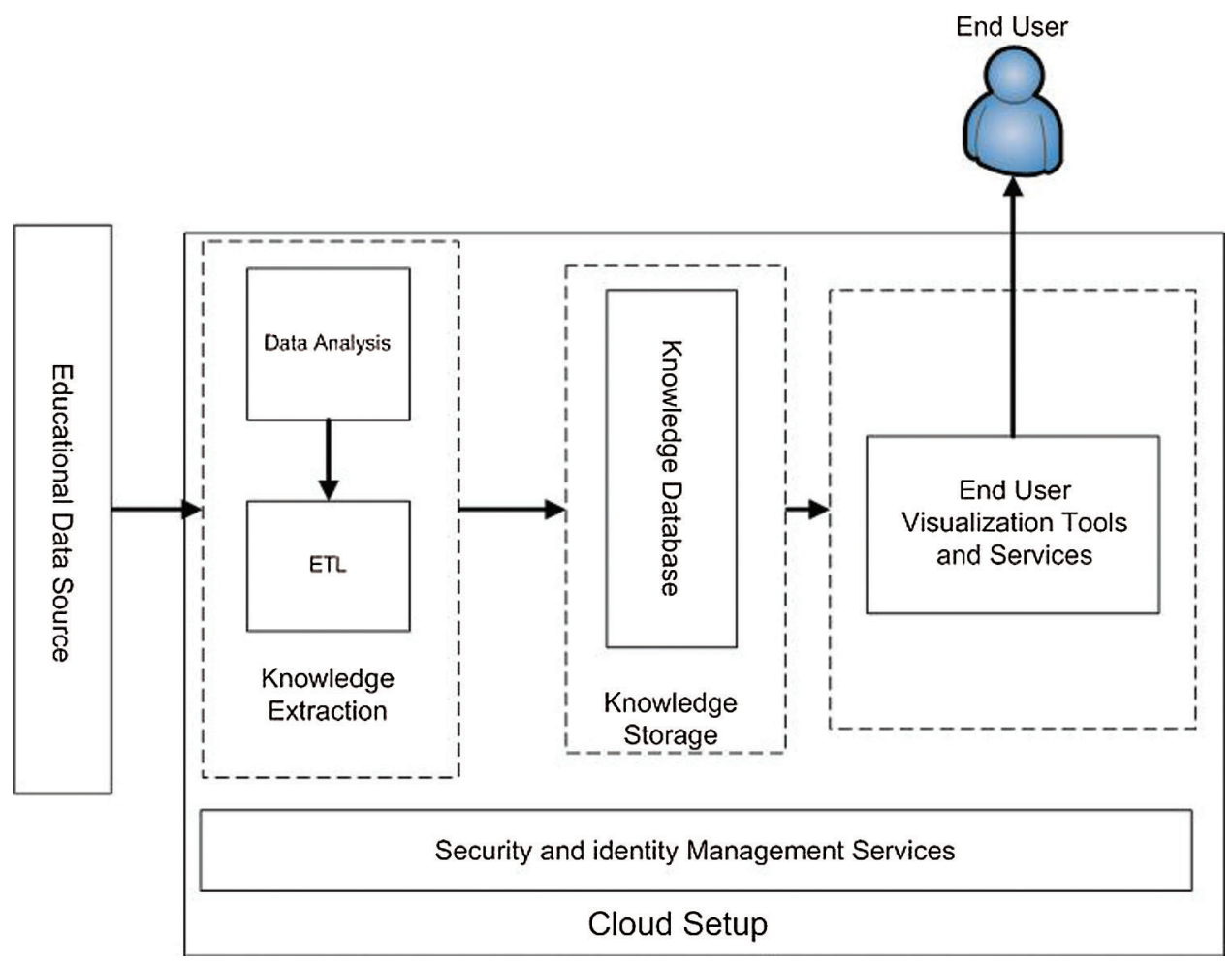

Figure 1: Cloud-based knowledge management framework for decision making in the education sector

The ETL process will formulate anomaly-free data into the required customized format. The result of the ETL will improve the analysis process by loading high-quality normalized data into a target storage. Fig. 2 illustrates the knowledge extraction from various sources of the educational setup.

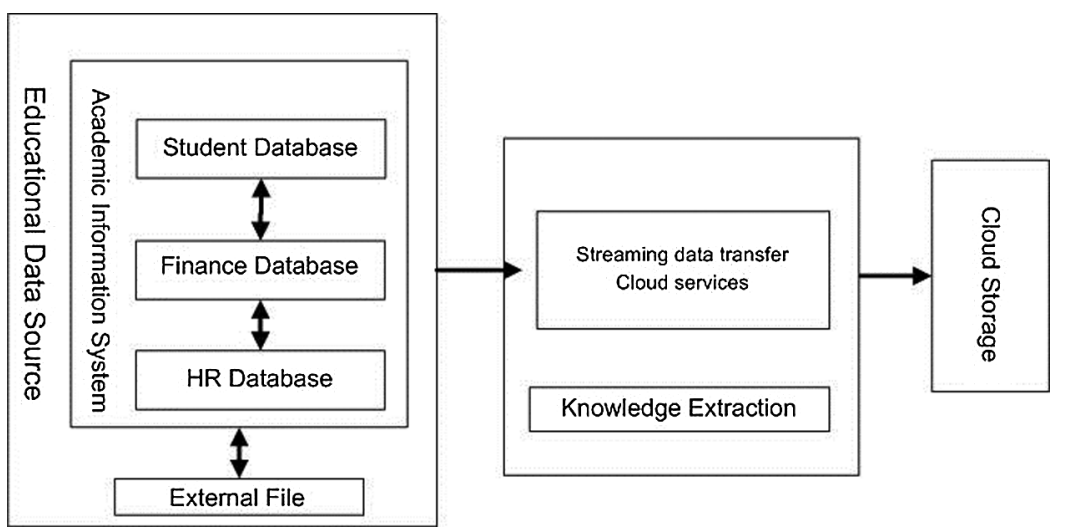

Figure 2: Knowledge extraction in the educational setup

As discussed earlier, knowledge is extracted to meet the decision-making purpose and sharing purpose only, and is intended to provide high-level information to end-users. Therefore, there is a need to transfer the selected information over the cloud rather than the whole database to minimize the security risk associated with the cloud. For data synchronization purposes, lift and shift Azure jobs can either be applied or one can create their ETL to perform this activity. Organizations can also use cloud services to make use of the ETL 
process. Several cloud services support ETL processes at different levels based on the data and organizational needs. Tab. 4 highlights the list of important service providers that can be used to perform ETL in an academic setup.

Table 4: List of cloud services for data extraction in the educational sector

\begin{tabular}{ll}
\hline Cloud Service Provider & Data Extraction Service \\
\hline Amazon & Amazon Kinesis \\
Google & Cloud Pub/Sub \\
Microsoft & Data Factory, power query \\
\hline
\end{tabular}

\subsection{Knowledge Storage}

The knowledge extracted in phase one is stored in cloud storage to perform further actions and processing. The suitable cloud service can be applied for knowledge storage depending upon the type and nature of the extracted information.

Many service providers offer storage over the cloud with SQL and NoSQL data from an educational setup. The proposed framework uses the Microsoft Azure Data Lake Store to accomplish the knowledge storage phase. Tab. 5 lists the data storage service from a top cloud service provider with the type of storage.

Table 5: List of cloud services for data storage in the educational sector

\begin{tabular}{ll}
\hline Service Provider & Data Storage Service \\
\hline Amazon & Amazon (S3) Simple Storage Service, Amazon Glacier \\
Google & Cloud SQL, Cloud Storage \\
Microsoft & Data Lake Store, Blob Storage, Azure Storage Account \\
\hline
\end{tabular}

\subsection{End-User Visualization Tools and Services}

Once the required knowledge is loaded on the cloud, the visualization tool will be applied to format the data according to end-user needs coupled with a powerful analytical tool that is capable of converting information into a graphical and statistical tabular format.

The outputs are the managerial reports used for decision-making purposes as well as for using inter and intra knowledge sharing tasks. Fig. 3 illustrates the cloud service for the visualization tools in the framework. Power BI is used in the proposed framework for end-user visualization since it supports a Business intelligence tool which is an ideal platform for decision-making purposes. Tab. 6 lists the top service providers that support the visualization tools for end-users in the education setup.

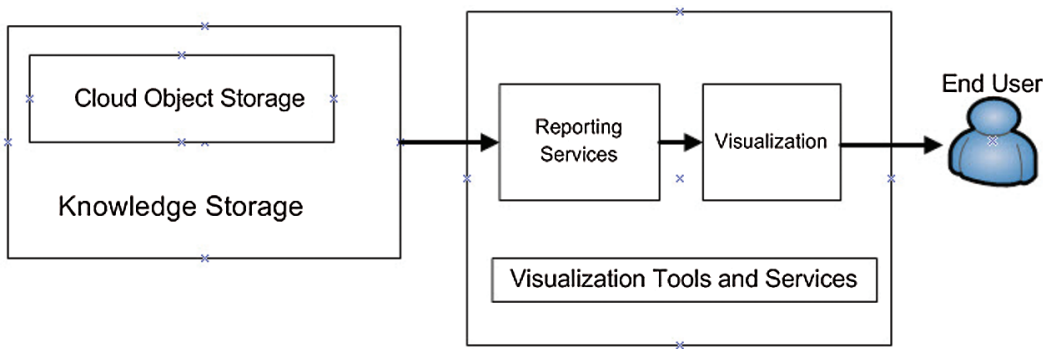

Figure 3: End-user visualization tools and services over the cloud 
Table 6: List of cloud service for end-user visualization

\begin{tabular}{ll}
\hline Service Provider & Visualization service \\
\hline Amazon & Amazon QuickSight \\
Google & Google Data Studio, Cloud Data Lab \\
\hline
\end{tabular}

\subsection{End-User}

Top-level administrators, deanships, faculties and other educational institution representatives can be the end-users who can access and use the applications based on the rights and privileges granted to each one preauthenticated by the application administrator's post approval from the concerned manager. Knowledge sharing is a key factor for the KMS and the proposed framework allowing knowledge to be shared with the users by offering role-based access.

\section{Reference Architecture For KSA Public University}

The university's Academic Affairs unit is responsible for all academic-related activities including teaching load management, staff relocation, management to make strategic decisions and other planning activities. Fig. 4 illustrates the reference architecture of Jazan University KSA. In this architecture, Power BI is deployed over the cloud using the PaaS deployment model. A built-in power query is used to perform the ETL to extract the knowledge from the source data. Depending upon the need, data analysis is performed for proper implementation of business logic to meet the end-user requirements. The knowledge extracted in the first stage is to be saved in the knowledge DB overcloud. A full data synchronization process is applied using Power Gateway to update the information with the source system.

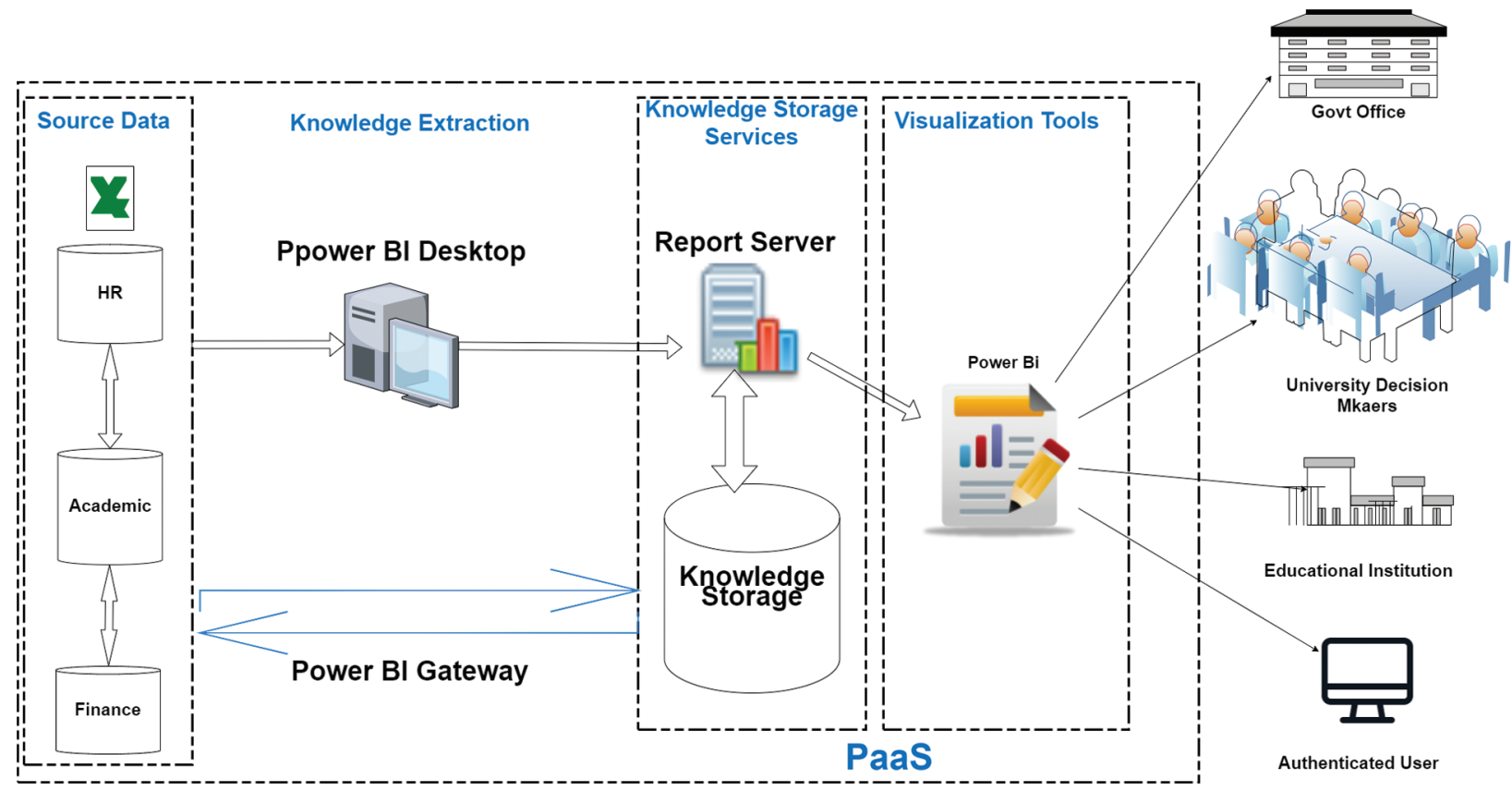

Figure 4: Cloud-based knowledge management for decision making in Jazan University KSA 
Power BI is used for end-user visualization which generates customized reports that produce comprehensive information to be used for decision-making purposes and other managerial activities. It also generates statistical reports intended for different management decision purposes at different levels. It allows user-defined customized query and report generation to be used in different decision-making and knowledge sharing practices as per the management needs.

\section{Result and Discussion}

The proposed cloud-based KM decision-making framework currently built for a university setting is based on the above methodology and implemented as a prototype in Jazan University KSA as shown in Fig. 4. The purpose of the prototype is to simulate the objectives and representation of solving the problem from the decision-maker's perspective. The results strongly allude to the fact that there is a significant requirement for the full implementation of a cloud-based KM decision-making framework in the university setting. To verify the results and validate the system, the university staff's, including the executives and decision-makers, feedback and system rating are taken into account. We have discussed in detail the key result factors for both the conventional and proposed processes in the section below.

\subsection{Performance in Terms of Data}

Data is the key information source and all the processes are also extracted data-dependent. Henceforth, data collation coupled with knowledge inference from the extracted data must meet the users' requirements with efficiency and efficacy. Fig. 5 illustrates a comparison between the proposed and existing conventional processes concerning different data characteristics. Both groups $\mathrm{A}$ and $\mathrm{F}$ had been engaged to evaluate the proposed process based on the below characteristics. The result shows that the proposed process is more efficient concerning performance and efficacy.

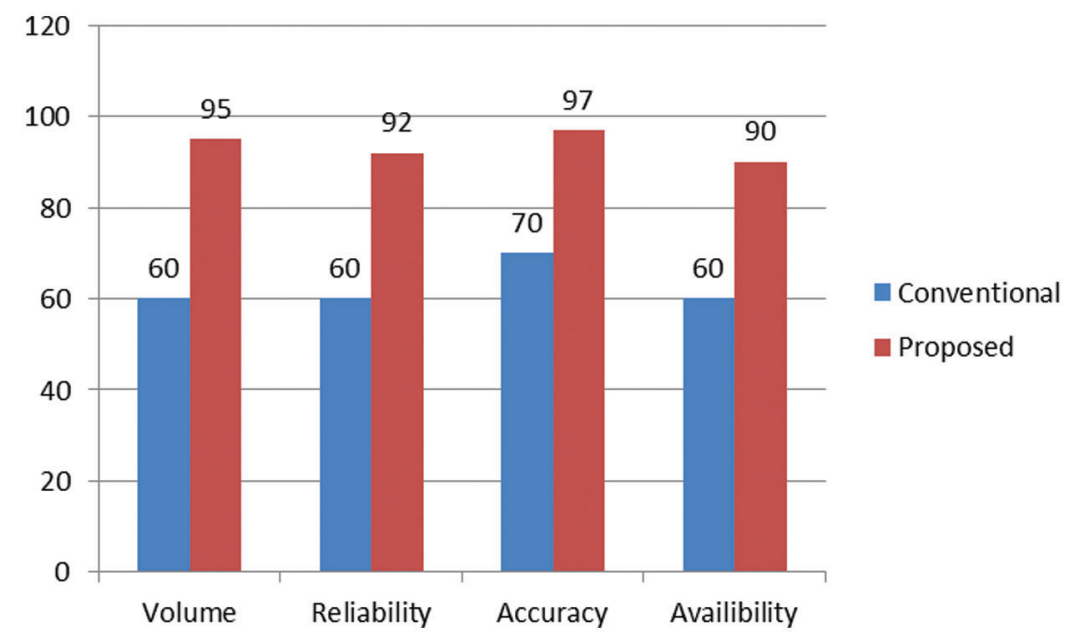

Figure 5: Data performance for conventional vs. proposed

\subsubsection{Volume}

This process focuses on the data collation in terms of volume. Further, it also describes the data collation approach for decision-making processing. The current data collation method is a combination of a manually operated and conventional information system which receives data from respective faculty members. This approach makes this activity very arduous and a time taking endeavor. However, the same process can be performed efficiently and rapidly by using the proposed framework which utilizes live data extraction. 


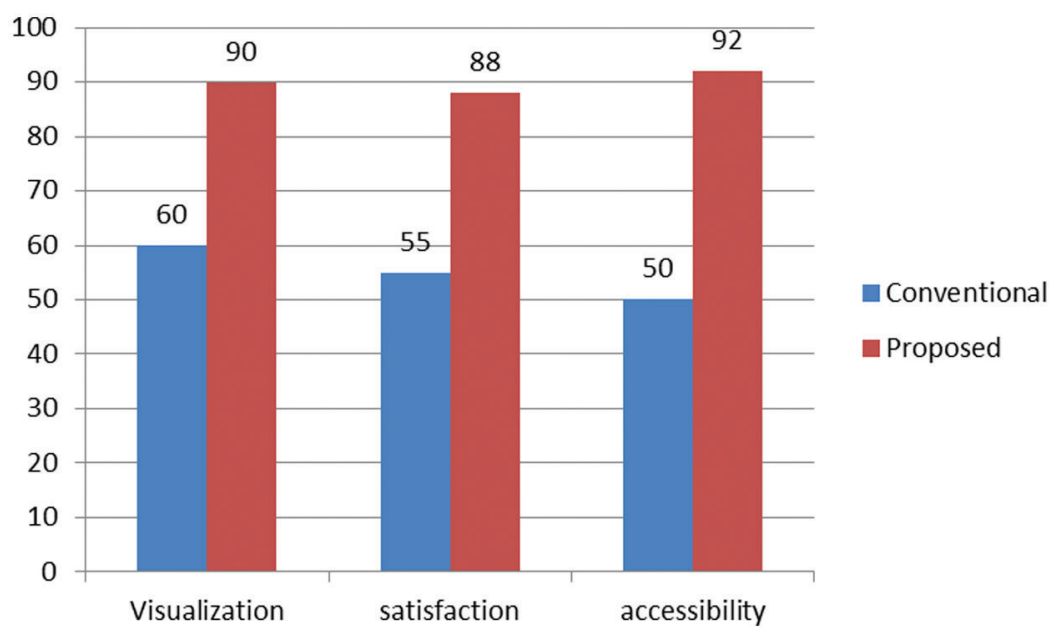

Figure 6: User performance for conventional vs. proposed

\subsubsection{Reliability}

How reliable is the data through the conventional process? The likelihood of human error is significant during entering the data being manipulated in the conventional process which creates data reliability concerns. Conversely, live data extraction is rather highly reliable, comparatively, and the proposed framework addresses these issues and concerns efficiently and reliably.

\subsubsection{Accuracy}

How accurate is the data corresponding to the latest alteration? Highly accurate information leads to an accurate and sound decision. Data processed by a conventional system needs to be updated periodically and the latest update would not be reflected in the existing database since it takes a while for updated alterations to appear. The proposed framework has the capability of producing accurate data due to its ability to provide live data extraction in comparison to the conventional process.

\subsubsection{Availability}

Statistical information is used by top management for decision-making purposes; on-demand information availability is the critical success factor in making a sound decision-making process. The proposed system achieves this critical factor by providing ubiquitous data availability.

\subsection{Performance in Terms of Effort}

Time is of key essence and a critical success factor in any process. Hence, the process which involves the least human intervention backed by deployment of the latest advanced technology will yield highly accurate and speedy results within a quick turnaround time.

Group F was engaged to evaluate the effort performance. The chart results in Fig. 6 shows a comparison between the proposed and conventional processes in terms of effort.

\subsubsection{Duration}

How long does it take to complete the whole processing cycle? This includes data collation time, data analysis, data process, and end-user perception and visualization. The current process takes a long time for data collation and processing which prolongs the overall duration of the process. The proposed framework eliminates conventional data collation methods and processing, and significantly shortens processing times. 


\subsubsection{Man Power}

How much manpower is required to engage in process accomplishment? The conventional process involves human engagement at the data collation, processing and visualization stages implying a significant number of manpower resource deployments. The proposed process in the proposed framework phenomenally reduces the manpower resources. Tab. 7 highlights the manpower with task nature with engagement duration.

Table 7: Human engagement and time performance for conventional vs. proposed

\begin{tabular}{llll}
\hline Process & Man Power & Task Nature & Completion Duration(Days) \\
\hline Conventional & 10 & Administrative task & 35 \\
Proposed & 3 & Administrative task & 5 \\
\hline
\end{tabular}

\subsection{Overall Performance}

The end-user is the key system stakeholder and user evaluation is the key success of the process. The main key indicator mentioned below describes the comparison of both the proposed and conventional processes to evaluate the overall performance in terms of users. Group A was engaged to evaluate the result concerning the main stockholder of the system. The results in Fig. 6 demonstrate a comparison of both processes.

\subsubsection{Visualization}

How much knowledge is realized and visualized corresponding to the decision reports? This spans the use of visualization tools to present an appropriate graphical representation to suitably meet end-user needs.

\subsubsection{Satisfaction}

What is the end user's satisfaction factor corresponding to the final output? It encompasses overall user satisfaction related to the outcomes, performance, intuitiveness and reliability of the information output.

\subsubsection{Accessibility}

How is the data accessible to the university's internal and external entities? It spans the on-demand latest data available as and when required.

Fig. 6 illustrates a comparison between the proposed and existing conventional processes concerning different data characteristics.

\section{Challenges and Future Research}

A cloud-based setup is an ideal and most affordable solution for any institution considering its adoption. This solution enables the HEIs to stay abreast with the latest technological and digital trends.

Despite the cloud's security robustness and low-security breach feature, it is still a concern and poses a barrier to shift on to the cloud due to data sensitivity. All the academic institutions prefer operating in a private cloud environment setup over a public cloud even though it is costly and requires an independent setup compared to the public. This is also a challenge in itself to convince them to shift over to the public cloud instead due to low operating costs. Moreover, the selection of appropriate visualization applications for end-users also falls in the same category. The proposed framework is limited to administrative staff and top-level decision-makers only. Student access is not included in the system in the proposed framework. This will be endeavored in the future expansion of the framework. 


\section{Conclusion}

Knowledge Management is an essential pillar for university management for effective decision making. It enables the top-level management to successfully making effective decisions based on the availability of reliable and updated information. The state-of-the-art concept of the latest cloud-based knowledge management is luring many organizations and institutions to shift to it owing to its immense advantages. The proposed framework is supposed to be the latest contribution in Cloud-based Knowledge Management literature. This study concludes with the proposal and design of an efficient framework for knowledge-based decision-making in a cloud environment. Since the universities being the main stakeholders of HEIs, they always strive to acquire sufficient skills to stay abreast and ahead in the academic business. Another added feature of CC enables capturing a faculty's accomplishments and achievements which allows the decision-makers to judge their professional growths and promotions. The results raise the staff confidence levels and possibly offer job security based on performance.

The decision-makers require quality of information rather than volume of data. The proposed framework provides exactly that by extracting the required information and presenting it in a statistical form for the toplevel management decision-making process. It reduces data extraction processing time by scheduling data synchronization with source data thereby assuring on-demand and updated knowledge availability. The core activity of the proposed framework is knowledge sharing that allows knowledge to be shared with end-users and other universities using role-based access.

For this scholastic endeavor, we have constructed a reference architecture from one of the public universities in KSA. The prototype results based on user feedback are promising and, thus, encourage its full implementation. The requirements can be general for any other university across KSA, but the implementation process is subject to vary based on ground realities that require further research currently beyond the scope of this study.

Funding Statement: The authors received no specific funding for this study.

Conflicts of Interest: The authors declare that they have no conflicts of interest to report regarding the present study.

\section{References}

[1] C. Wang, C. Zhao and L. Zhang, "A framework for management of massive knowledge in cloud environment," in Biomedical Engineering and Informatics (BMEI), 2014 7th Int. Conf., Dalian, China, pp. 843-847, 2014.

[2] S. M. Noor, M. Younas and M. Arshad, "A review on cloud based knowledge management in higher education institutions," International Journal of Electrical \& Computer Engineering, vol. 9, no. 6, pp. 5420-5427, 2019.

[3] M. Alam and K. A. Shakil, "Cloud database management system architecture," UACEE International Journal of Computer Science and its Applications, vol. 3, no. 1, pp. 27-31, 2013.

[4] C. Stergiou and K. E. Psannis, "Efficient and secure BIG data delivery in Cloud computing," Multimedia Tools and Applications, vol. 76, no. 21, pp. 22803-22822, 2017.

[5] P. Nilsook Anupan and P. Wannapiroon, "A framework for a knowledge management system in a cloud computing environment using a knowledge engineering approach," International Journal of Knowledge Engineering, vol. 1, no. 2, pp. 146-149, 2015.

[6] I. Chikhi and H. Bouarfa, "Knowledge management process through a cloud computing based approach," in European Conf. on Knowledge Management, Lisbon, Portugal, pp. 238-247, 2019.

[7] M. P. Rahamathulla, "Cloud-based healthcare data management framework," KSII Transactions on Internet \& Information Systems(TIIS), vol. 14, no. 3, pp. 1014-1025, 2020.

[8] A. Alhammadi, "A knowledge management based cloud computing adoption decision making framework," Ph.D. dissertation. Staffordshire University, United Kingdom, 2016. 
[9] M. Al-Qurishi, M. Al-Rakhami, M. AlRubaian and A. Alamri, "A framework of knowledge management as a service over cloud computing platform," in Proc. of the Int. Conf. on Intelligent Information Processing, Security and Advanced Communication, Batna, Algeria, pp. 10, 2015.

[10] C. N. Liao, I. Chih and Y. K. Fu, "Cloud computing: A conceptual framework for knowledge management system," Human Systems Management, vol. 30, no. 3, pp. 137-143, 2011.

[11] M. F. Baharuddin, T. A. T. Izhar, A. N. Mohamad and W. Hasnol, "A framework based knowledge management system (KMS) for dynamic decision-making (DDM)," International Journal of Academic Research in Business and Social Sciences, vol. 6, no. 4, pp. 287-294, 2016.

[12] J. R. de Oliveira, S. F. Stefenon, C. K. Yamaguchi, A. C. R. Klaar and M. J. Sembay, "How to improve decision making knowledge management," International Journal of Development Research, vol. 7, no. 9, pp. 15279-15282, 2017.

[13] K. M. Masum, L. S. Beh, M. A. K. Azad and K. Hoque, "Intelligent human resource information system (i-HRIS): A holistic decision support framework for HR excellence," International Arab Journal of Information Technology, vol. 15, no. 1, pp. 121-130, 2018.

[14] J. Feldman, Decision Management as Continuing System Education, 2015. [Online]. Available: https://www. linkedin.com/pulse/decision-management-continuing-system-education-jacob-feldman/?trk=mp-reader-card.

[15] E. A. Ahmed and H. A. Ahmed, "A proposed model for education system using cloud computing," in 2018 3rd Int. Conf. on Emerging Trends in Engineering, Sciences and Technology (ICEEST), Karachi, Pakistan, pp. 1-4, 2018.

[16] N. J. Navimipour, A. M. Rahmani, A. H. Navin and M. Hosseinzadeh, "Expert cloud: A cloud-based framework to share the knowledge and skills of human resources," Computers in Human Behavior, vol. 46, pp. 57-74, 2015.

[17] M. Rafiq, A. Bashar and A. Shaikh, "Innovative trends in knowledge management: A cloud computing perspective," in Proc. of the First Middle East Conf. on Global Business, Economics, Finance and Banking, Dubai, 2014.

[18] P. Raj, "Knowledge management—A road map for winning organization," International Journal of Research in Economics \& Social Sciences, vol. 2, no. 2, pp. 363-373, 2012.

[19] A. Anand and M. Singh, "Understanding knowledge management," International Journal of Engineering Science and Technology, vol. 3, no. 2, pp. 926-939, 2011.

[20] V. S. Anantatmula, "Designing meaningful KM processes to improve organizational learning," Trends in Information Management (TRIM), vol. 5, no. 2, 2012.

[21] W. Q. Qwaider, “Integrated of knowledge management and E-learning system," International Journal of Hybrid Information Technology, vol. 4, no. 4, pp. 59-70, 2011.

[22] M. M. Amine and M. Ahmed-Nacer, "An agile methodology for implementing knowledge management systems: A case study in component-based software engineering," International Journal of Software Engineering \& Applications, vol. 5, no. 4, pp. 159-170, 2011.

[23] H. Ramayani, G. Wang, H. Prabowo, T. Sriwidadi, R. Kodirun et al., "Improving organizational knowledge management (KM) through cloud based platform in higher education," in 2017 Int. Conf. on Information Management and Technology (ICIMTech), Yogyakarta, Indonesia, pp. 10-13, 2017.

[24] Z. H. Luo, L. X. Yun and X. Zhang, "Cloud computing-based KMS in comprehensive hospitals," in Proc. of the 2014 Int. Conf. on Management, Information and Educational Engineering (MIEE 2014), Xiamen, China, pp. 361, 2015.

[25] S. Kale, "Cloud computing—Types of cloud," 2018. [Online]. Available: https://www.esds.co.in/blog/cloudcomputing-types-cloud/\#sthash.4Jhcx7fb.dpbs.

[26] H. Gangwar, H. Date and R. Ramaswamy, "Understanding determinants of cloud computing adoption using an integrated TAM-TOE model," Journal of Enterprise Information Management, vol. 28, no. 1, pp. 107-130, 2015.

[27] S. T. Ismail, "The role of marketing information system on decision making an applied study on Royal Jordanian Air Lines (RJA)," International Journal of Business and Social Science, vol. 2, no. 3, 2011.

[28] I. Litvaj and D. Stancekova, "Decision-making, and their relation to the knowledge management, use of knowledge management in decision-making," Procedia Economics and Finance, vol. 23, pp. 467-472, 2015. 
IASC, 2022, vol.31, no.1

[29] B. Delibašić and M. Suknović, "Loan granting knowledge system," Journal of Decision Systems, vol. 15, no. 2-3, pp. 309-329, 2006.

[30] K. A. Shakil, S. Sethi and M. Alam, "An effective framework for managing university data using a cloud based environment," in 2015 2nd Int. Conf. on Computing for Sustainable Global Development (INDIACom), New Delhi, India, pp. 1262-1266, 2015.

[31] J. Chen, "Framework of SOA-based equipment maintenance knowledge management system," in 2010 5th Int. Conf. on Computer Science \& Education, Hefei, China, pp. 912-917, 2010.

[32] Q. Duan, Y. Yan and A. V. Vasilakos, "A survey on service-oriented network virtualization toward convergence of networking and cloud computing," IEEE Transactions on Network and Service Management, vol. 9, no. 4, pp. 373-392, 2012.

[33] M. Oprea, "A university knowledge management tool for academic research activity evaluation," Informatica Economica, vol. 15, no. 3, pp. 58, 2011.

[34] M. F. Baharuddin, M. N. Masrek and M. S. M. Shoid, "Information dissemination strategies among migrant workers: A conceptual framework," International Journal for Infonomics (IJI), vol. 8, no. 3, 2015.

[35] R. Kimball and J. Caserta, "The data warehouse ETL toolkit," John Wiley \& Sons, 2004. [Online]. Available: http://data.theeuropeanlibrary.org/BibliographicResource/1000098306979.

[36] J. Runtuwene, I. R. Tangkawarow, C. Manoppo and R. Salaki, "A comparative analysis of extract, transformation and loading (ETL) process," in IOP Conf. Series: Materials Science and Engineering, Manado, Indonesia, 2018. 